\title{
GLOBAL DISK OSCILLATIONS: THEORETICAL LINE PROFILES
}

\author{
W. HUMMEL* and R.W. HANUSCHIK \\ Astronomisches Institut, Ruhr-Universität Bochum, D-44780 Bochum, Germany
}

\begin{abstract}
Based on 3D radiative line transfer calculations we present $\mathrm{H} \alpha$ emission line profiles of Be star circumstellar envelopes undergoing one-armed global disk oscillations. The results are in agreement with the observed line profile variability.
\end{abstract}

\section{The model}

Kato (1983) and Okazaki (1991) proposed one-armed global disk oscillations (GDO) as an explanation for the long-term variability of optical Be star emission lines. The disk perturbation pattern is rotating retrograde around the $\operatorname{star}(P \simeq 10$ years $)$ giving rise to the observed long-term line profile variability (Hanuschik et al., 1993). The perturbed density distribution consists of a significant maximum region located nearby the central star at azimuthal angle $\phi=0^{\circ}$. The particle trajectories are similar to ellipses. Based on Okazaki's perturbation pattern and on a 3D radiative line transfer code (Hummel, 1993) we calculated $\mathrm{H} \alpha$ emission line profiles of circumstellar disks extended up to $R_{\mathrm{d}}=5 R_{*}$ with a radial density run of $N \sim N_{0}\left(\frac{r}{R_{*}}\right)^{-2}$.

\section{Results and discussion}

In Fig.1 and in Fig. 2 the line profile sequences of different values of $\phi$ simulate the observed long-term profile variability. At $\phi=0^{0}$ the line of sight is parallel to the symmetry axis of the GDO pattern and the maximum density region is in front of the star. At $\phi=90^{\circ}$ the maximum density region is on the left hand side of the star moving towards the observer.

At nearly pole-on view $\left(i=10^{\circ}\right)$ variations of asymmetric winebottle-type profiles are presented, at $i=30^{\circ}$, typical $V / R$-ratio variabilities of doublepeak profiles are shown and for an edge-on view $\left(i=90^{\circ}\right)$ the calculated shell profiles exhibit variations of the radial velocity $\left(V_{c d}\right)$ and of the flux $\left(F_{c d}\right)$ at the central depression of the profile. The observable sequence of $[V / R>1] \Rightarrow\left[F_{\mathrm{cd}}^{\min }\right] \Rightarrow[V / R<1] \Rightarrow\left[F_{\mathrm{cd}}^{\max }\right]$ (Cowley \& Gugula, 1973) is in agreement with a retrograde pattern rotation (Fig.2; $i=60^{\circ}$ ).

Acknowledgements: Many thanks go to J. Dachs and D. Baade for helpful discussions. Financial support by the Deutsche Forschungsgemeinschaft under grant $\mathrm{Da} 75 / 12-2$ is gratefully acknowledged. Plots were executed by means of ESO-MIDAS/91MAY.

* now at: Astrofysisch Instituut, Vrije Universiteit Brussel, Pleinlaan 2, B-1050 Brussel, Belgium 

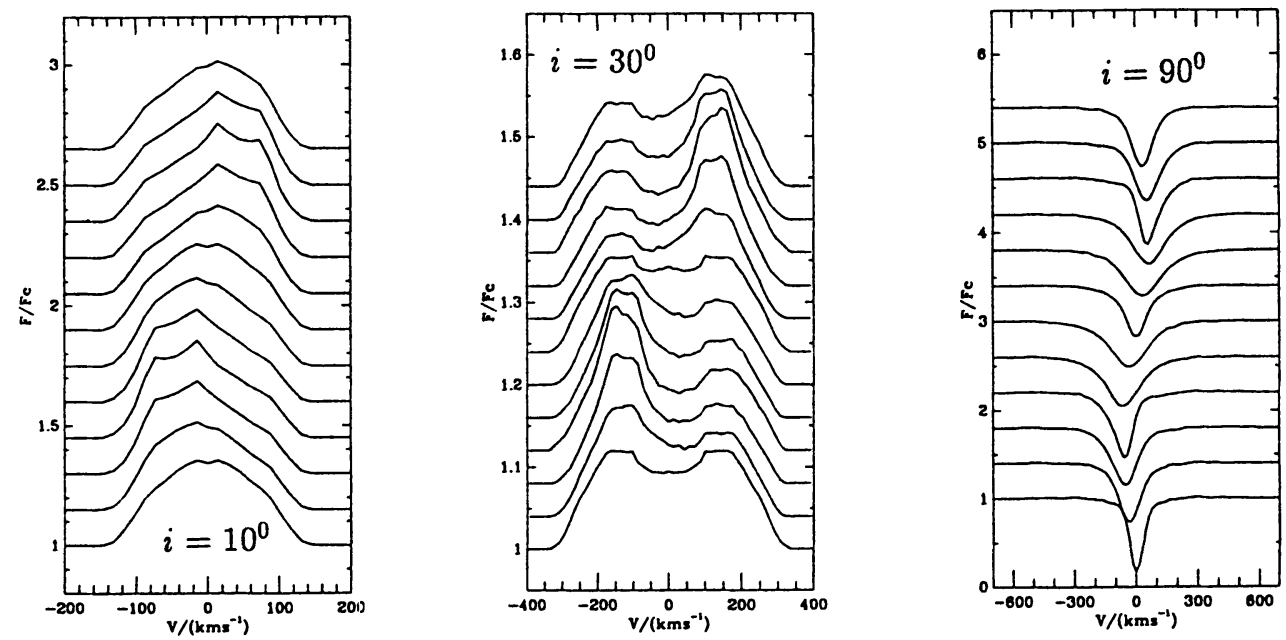

Fig. 1. Three sequences of calculated emission line profiles of a nearly Keplerian disk $\left(N_{0}=10^{13} \mathrm{~cm}^{-3}\right)$ at $i=10^{\circ}, i=30^{\circ}$ and $i=90^{\circ}$. In each sequence the azimutal angle $\phi$ varies from bottom to top: $\phi=0^{\circ}, 30^{\circ}, 60^{\circ}, \ldots 330^{\circ}$.
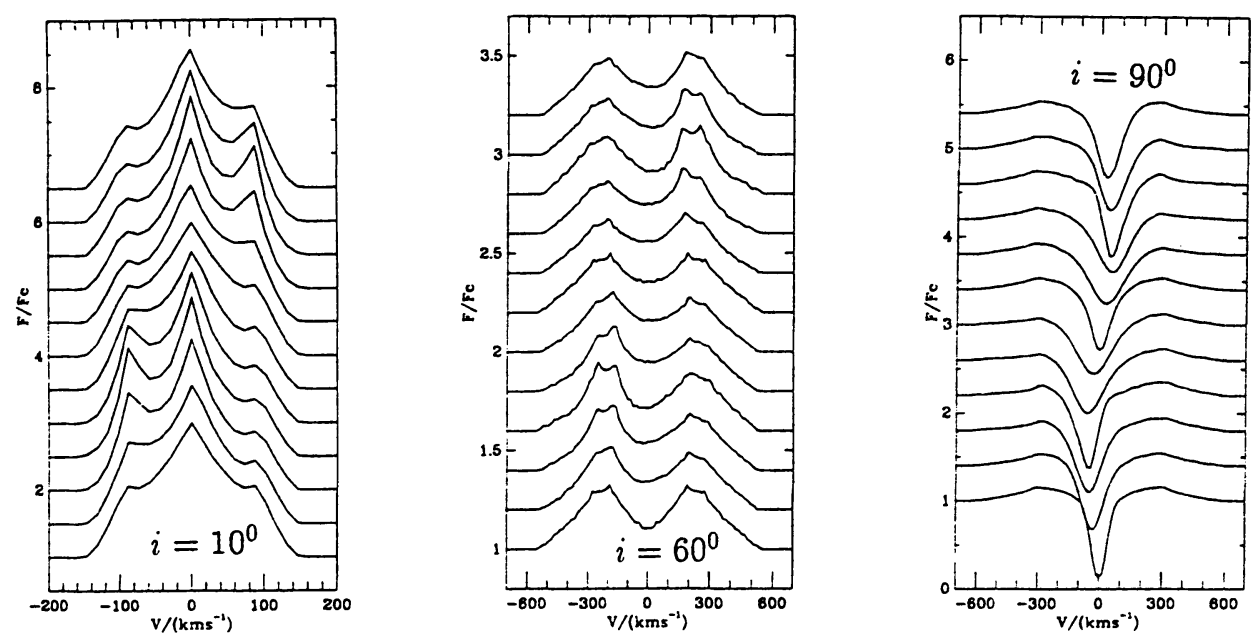

Fig. 2. As Fig.1, except $N_{0}=10^{14} \mathrm{~cm}^{-3}$ and $i=10^{\circ}, i=60^{\circ}$ and $i=90^{\circ}$.

\section{References}

Cowley, A., Gugula, E.: 1973, Astron. Astrophys. 22, 203

Hanuschik, R.W., Hummel, W., Dietle, O., Dachs, J., Sutorius, E.: 1993, these proceedings Hummel, W.: 1993, PhD., Bochum

Kato, S.: 1983, Publ. Astron. Soc. Japan 35, 24

Okazaki, A.T.: Publ. Astron. Soc. Japan 43, 75 